\title{
La Hidatidosis infantil en el Chile de hoy, un problema de desigualdad social
}

Paulina Martínez-Gallegos*

\begin{abstract}
*Doctora en Salud Pública. Universidad Diego Portales. Formación General. Santiago de Chile. Chile Correspondencia: Dra. Paulina Martinez Gallegos. Correo electrónico: paulinamartinezg@yahoo.com. Dirección: Peatones veinte 4577. Ñuñoa. Santiago. Chile. Código postal 7750000.
\end{abstract}

\section{RESUMEN}

Introducción: la hidatidosis en menores de edad representa una infección relativamente reciente, donde cada nueva infección significa el fracaso de las medidas preventivas y de control, donde a la vez se refleja una desigualdad en la presentación de esta infección. Objetivo: caracterizar y evaluar la desigualdad de la hidatidosis en menores entre o y 18 años de edad en Chile en el periodo 2001 al 2011. Materiales y método: se emplearon los datos nacionales de egresos hospitalarios para el cálculo de las tasas absolutas, diferencias de tasas y riesgos atribuibles. La desigualdad se evaluó con el Coeficiente de Gini. Como indicadores socioeconómicos se emplearon los PIB per capita regional (2008 al 2011) y el Índice de Desarrollo Humano regional (2003). Resultados: si se pudiesen igualar las condiciones socioeconómicas a nivel regional se podrían evitar entre 37 a 68 egresos hospitalarios extras por hidatidosis. Conclusiones: existe un impacto de la gradiente social sobre la presentación de esta enfermedad en menores de 18 años en Chile para el período en estudio. MÉD.UIS. 2017;30(2):29-36.

Palabras clave: Hidatidosis. Equinococcosis en menores. Enfermedad desatendida. Desigualdad.

\section{Child Hidatidosis in Chile today: a problem of social inequality}

\section{ABSTRACT}

Introduction: hydatid disease in minors represent relatively recent infections, with each new infection means the failure of preventive and control measures, where both an inequality is reflected in the presentation of this infection. Objective: to characterize and evaluate the inequality of hydatid disease in children aged o to 18 years in Chile in the period 2001 to 2011. Materials and Methods: hospital discharges for analysis of the rate ratio, rate difference, population attributable risk percentage and Gini coefficient were used. As economic indicators of regional GDP per capita (2008 to 2011) and the Regional Human Development Index 2003 were used. Results: if they could match the socio-economic conditions at the regional level could be avoided between 37 to 68 extra per hydatid disease hospital discharges. Conclusions: there is an effect of the social gradient on the presentation of this disease.in children under 18 years in Chile for the period under study. MÉD.UIS. 2017;30(2):29-36.

Keywords: Hydatidosis. Echinococcosis in children. Neglected disease. Inequality.

¿Cómo citar este artículo?: Gallegos-Martínez P. La Hidatidosis infantil en el Chile de hoy, un problema de desigualdad social. MÉD.UIS. 2017;30(2):29-36.

\section{INTRODUCCIÓN}

La hidatidosis es una enfermedad causada por el género Echinococcus granulosus, s. $^{1,2}$ Chile, esta antropozoonosis es la enfermedad parasitaria de mayor importancia, estando sujeta al Sistema de Enfermedades de Notificación Obligatoria (ENO), por generar altos costos socieconómicos y sanitarios, incluyendo gastos por diagnóstico, tratamiento, ausentismo laboral/escolar, hospitalizaciones, 
cirugías, y muertes prematuras, entre otros ${ }^{3,4}$. El parásito causal fue identificado en 1808 y su ciclo biológico en 1853 , sin embargo, a la fecha persiste como problema de salud pública particularmente alojado en poblaciones postergadas. La falta de comprensión de los diversos factores involucrados en el ciclo de transmisión-enfermedad y la ausencia de políticas públicas sostenidas en el tiempo son algunas de las razones asociadas a la presencia de esta zoonosis. Si bien el contagio ocurre a cualquier edad, es más fácil en los primeros años de vida dadas las costumbres de los menores de edad, lo cual contribuye a la ingestión de huevos desde las heces de los hospederos definitivos ${ }^{5}$.

Martínez (2017) indica que las estadísticas en Chile, en menores entre o y 18 años de edad, muestran una variación de casos, desde 79 en 2001 (58,2\% en hombres) a 18 en 2011 (50\% en hombres), con un promedio anual cercano a 66 casos. La tasa de notificación ha cambiado desde 1,48 casos por 100000 habitantes en 2001, a 0,36 casos por 100000 habitantes en 2011, reflejando una tendencia a la disminución. En relación a la edad, el grupo etario que presenta la mayor tasa de notificación corresponde entre los 5 y 9 años, con una tasa de 18,6 casos por 100000 habitantes entre o y 18 años ${ }^{6}$.

En Chile, los egresos hospitalarios se han modificado desde 236 en 2001 (58,1\% hombres) a 138 en 2011 ( $52,2 \%$ hombres), con un promedio anual cercano a 190 egresos y un total acumulado de 2089 (56,6\% hombres). La tasa de egresos hospitalarios se ha trasladado desde 4,4 a 2,8 egresos por 100000 habitantes, entre 0-18 años, con una tendencia al decrecimiento. Según tiempo de estadía, se registra una mediana de 8 días (1-120 días). Según la condición de egreso, el 95,5\% egresaron vivos y sólo el 4,5\% fallecidos. El análisis por sistema de previsión muestra que el $94,7 \%$ de los menores egresados son beneficiarios del Fondo Nacional de Salud (FONASA corresponde al subsistema público, que atiende a cerca del $80 \%$ de la población chilena).

En Chile, las defunciones alcanzan a 7 (mujeres $71,4 \%$ ), registrándose la última en el año 2008. Se ha de considerar que toda infección en menores de edad, particularmente entre o y 4 años se traduce en nuevas infecciones $y$, consecuentemente, en un fracaso de las medidas de prevención y control.

La desigualdad en salud es entendida como la distribución heterogénea de la salud, es decir, una variabilidad que puede responder a diferentes causas. Se ha de establecer que desigualdad no es sinónimo de inequidad. La inequidad es una desigualdad injusta y evitable, y consecuentemente como primer paso a la identificación de inequidades en salud es poder identificar desigualdades.

Este trabajo tiene como objetivo caracterizar y evaluar la desigualdad de la presentación de esta enfermedad en menores entre o y 18 años de edad en Chile. Para ello, se realizó una identificación del impacto del contexto socioeconómico y demográfico sobre la incidencia de casos de morbilidad (egresos hospitalarios) de hidatidosis en Chile, período 2001-2011.

\section{Materiales y método}

Se trata de un estudio descriptivo, empleando datos secundarios, recolectados de los egresos hospitalarios de Chile para el período 2001-2011, dada su mayor confiabilidad en su notificación. Como criterios de inclusión se consideraron todos los egresos hospitalarios diagnosticados con hidatidosis en menores de edad en Chile, durante el período de estudio.

Dentro de la caracterización del impacto del contexto socioeconómico y demográfico sobre la incidencia de casos de morbilidad de hidatidosis en Chile 20012011, se evaluó la desigualdad en la presentación de esta zoonosis.

Se emplearon los egresos hospitalarios de Chile para el análisis de la Razón de Tasas (RT), Diferencia de Tasas (DT), Riesgo Atribuible Poblacional Porcentual (RAP\%) y Coeficiente de Gini. Como indicadores o variables socioeconómicas se emplearon el Producto Interno Bruto (PIB) per capita regional para los años disponibles 2008 al 2011 y el Índice de Desarrollo Humano (IDH) ${ }^{* *}$ regional, para el cual sólo se disponía de datos para el año 2003. Para los diferentes análisis se emplearon los programas Excel, SPSS 10.0 y Stata 12.0.

Razón (RT) y Diferencia de Tasas (DT): estas medidas permitieron comparar dos regiones con tasas de morbilidad extremas y responder a preguntas como ¿Cuántos egresos hospitalarios hay en la región de Chile con menores indicadores socioeconómicos en relación a la región chilena con mejores indicadores? y ¿Cuántos egresos hospitalarios significa esto en números absolutos?. Se utilizaron las tasas de egresos hospitalarios 
mAYO - AGOSTO

regionales para estimar la RT, para el PIB (obtenido desde el Banco Central) y el IDH (indicador con mirada multidimensional, que abarca aspectos de la vida bajo las dimensiones de salud, educación e ingresos. Lo anterior se fundamente en que las personas deben tener una vida sana y larga; poseer los conocimientos necesarios para comprender y relacionarse reflexivamente con el entorno social; y poseer los ingresos suficientes para acceder a un nivel de vida decente.

$\mathrm{RT}=$ Tasa de Egreso hospitalario de la región con menor $\mathrm{PIB}^{{ }^{*}}$

Tasa de Egreso hospitalario de la región con mayor PIB

La DT fue la diferencia entre la región de Chile con la situación económica más desfavorecida y la región chilena con mejor nivel. Luego, se llevó a números absolutos multiplicando el resultado obtenido en la diferencia de tasas por el total de la población de la región desfavorecida para cada año que se obtiene información?.

En este análisis se incluyen todas las regiones del país que presentan registros de egresos hospitalarios por hidatidosis.

Riesgo Atribuible Poblacional porcentual (RAP\%): esta medida de impacto permitió estimar la proporción de la tasa general de egresos hospitalarios que es posible disminuir si todos los grupos tuviesen las tasas de la región con el mejor nivel socioeconómico o con la menor tasa de egresos hospitalarios (considerados como grupos de referencia) $)^{7-10}$.

RAP\% = TEH país - TEH regional con la mejor situación x 100

$$
\text { TEH país }
$$

RAP\%= TEH país - TEH regional con la menor mortalidad $\times 100$

$$
\text { TEH país }
$$

Donde TEH es la Tasa de egresos hospitalarios.

Coeficiente de Gini: utilizado para medir cualquier forma de distribución desigual, sus valores van entre o y 1. Donde o se interpreta como la ausencia de desigualdad y el valor 1 como la máxima desigualdad ${ }^{8}$. El resultado donde no existen diferencias se conoce como equi-distribución; y la curva (Curva de Lorenz) hacia la esquina inferior derecha indica una alta desigualdad en el egreso hospitalario. Éste fue estimado mediante la fórmula de Brown.
Dentro de las variables utilizadas se encuentran las variables socioeconómicas expresadas a través del Producto Interno Bruto Regional (PIBR) per cápita 2008-2011 (Banco Central de Chile) y del nivel de desarrollo comunal mediante el Índice de Desarrollo Humano 2003 del Programa de Desarrollo de las Naciones Unidas (PNUD).

Tabla 1: PIBR per cápita, 2008- 2011

\begin{tabular}{|l|c|c|c|c|}
\hline \multicolumn{1}{|c|}{ Región } & $\begin{array}{c}\text { Per } \\
\text { cápita } \\
\mathbf{2 0 0 8}\end{array}$ & $\begin{array}{c}\text { Per } \\
\text { cápita } \\
\mathbf{2 0 0 9}\end{array}$ & $\begin{array}{c}\text { Per } \\
\text { cápita } \\
\mathbf{2 0 1 0}\end{array}$ & $\begin{array}{c}\text { Per } \\
\text { cápita } \\
\mathbf{2 0 1 1}\end{array}$ \\
\hline Arica y Parinacota & 3,2 & 3,0 & 3,2 & 3,5 \\
\hline Tarapacá & 9,5 & 9,5 & 9,2 & 8,5 \\
\hline Antofagasta & 17,8 & 17,4 & 17,9 & 16,5 \\
\hline Atacama & 7,0 & 6,8 & 7,9 & 8,7 \\
\hline Coquimbo & 3,7 & 3,4 & 4,0 & 4,2 \\
\hline Valparaíso & 4,3 & 4,1 & 4,2 & 4,4 \\
\hline $\begin{array}{l}\text { Región } \\
\text { Metropolitana de } \\
\text { Santiago }\end{array}$ & 6,0 & 5,9 & 6,2 & 6,6 \\
\hline $\begin{array}{l}\text { Libertador } \\
\text { General Bernardo } \\
\text { O'Higgins }\end{array}$ & 4,4 & 4,6 & 4,6 & 4,9 \\
\hline Maule & 2,9 & 3,0 & 3,0 & 3,3 \\
\hline Biobío & 3,4 & 3,4 & 3,4 & 3,8 \\
\hline La Araucanía & 2,0 & 1,9 & 2,1 & 2,2 \\
\hline Los Ríos & 2,7 & 2,7 & 2,9 & 3,2 \\
\hline Los Lagos & 2,9 & 2,7 & 2,6 & 2,9 \\
\hline $\begin{array}{l}\text { Aisén del General } \\
\text { Carlos Ibáñez del } \\
\text { Campo }\end{array}$ & 3,8 & 3,9 & 3,9 & 4,3 \\
\hline $\begin{array}{l}\text { Magallanes y de la } \\
\text { Antártica Chilena }\end{array}$ & 4,9 & 5,0 & 5,1 & 5,1 \\
\hline
\end{tabular}

Fuente: Banco Central de Chile, 2015

\section{RESULTADOS}

\section{RT Y DT SEGÚN PIB PER CAPITA REGIONAL}

La Tabla 3 muestra el resumen de las estimaciones de RT, DT y números absolutos de egresos hospitalarios según el PIB per capita regional, en menores entre 0-18 años de edad.

En el análisis según la RT, la región chilena consistentemente con menor PIB regional per capita fue la región de La Araucanía y la de mayor PIB fue la región de Antofagasta, seguida por la Región Metropolitana. 
Para el 2008 la RT indica que en la región chilena de menor PIB -o situación económica, correspondiente a la Región de La Araucanía, egresan por hidatidosis casi 33 veces más niños que en la Región Metropolitana, la cual dispone de mayor PIB (se utilizó esta región como comparación dado que en la región de Antofagasta -la de mayor PIB- no hubo egresos hospitalarios para este año). A la vez, la DT entre estas dos regiones fue de 11,1 por 100000 habitantes entre o y 18 años, lo cual significa en números absolutos, que en la región de La Araucanía hubo 68 egresos más en menores entre o y 18 años que los que podrían esperarse si su situación mejorase hasta el nivel de la Región Metropolitana.

Tabla 2: Índice de Desarrollo Humano Regional, 2003

\begin{tabular}{|c|c|c|c|c|}
\hline Región & $\begin{array}{c}\text { Índice } \\
\text { dimensión } \\
\text { salud }\end{array}$ & $\begin{array}{c}\text { Índice } \\
\text { dimensión } \\
\text { educación }\end{array}$ & $\begin{array}{c}\text { Índice } \\
\text { dimensión } \\
\text { ingreso }\end{array}$ & $\begin{array}{c}\text { IDH } \\
\text { regional }\end{array}$ \\
\hline Tarapacá & 0,784 & 0,771 & 0,637 & 0,731 \\
\hline Antofagasta & 0,764 & 0,764 & 0,660 & 0,729 \\
\hline Atacama & 0,804 & 0,753 & 0,591 & 0,716 \\
\hline Coquimbo & 0,812 & 0,725 & 0,615 & 0,717 \\
\hline Valparaíso & 0,779 & 0,764 & 0,614 & 0,719 \\
\hline O'higgins & 0,756 & 0,714 & 0,597 & 0,689 \\
\hline Maule & 0,744 & 0,696 & 0,585 & 0,675 \\
\hline Bío Bío & 0,749 & 0,724 & 0,585 & 0,686 \\
\hline Araucanía & 0,744 & 0,720 & 0,573 & 0,679 \\
\hline Los Lagos & 0,732 & 0,709 & 0,602 & 0,681 \\
\hline Aisén & 0,731 & 0,737 & 0,665 & 0,711 \\
\hline Magallanes & 0,732 & 0,772 & 0,695 & 0,733 \\
\hline Metropolitana & 0,801 & 0,774 & 0,704 & 0,760 \\
\hline País & 0,776 & 0,748 & 0,650 & 0,725 \\
\hline
\end{tabular}

Fuente: Programa de Desarrollo de las Naciones Unidas (PNUD) "Las trayectorias del Desarrollo Humano en las comunas de Chile (1994-2003)"

Tabla 3. Razón de Tasas, Diferencia de Tasas y Número Absoluto de egresos de niños 0 a 18 años por hidatidosis según PIB per cápita regional, 2008-2011

\begin{tabular}{|c|c|c|c|}
\hline Año & RT & DT & ${ }^{*} \mathbf{N}^{\circ}$ \\
\hline 2008 & 32,7 & 11,1 & 68,3 \\
\hline 2009 & 27,6 & 14,7 & 44,3 \\
\hline 2010 & - & 16,1 & 48,0 \\
\hline 2011 & - & 12,5 & 37,0 \\
\hline
\end{tabular}

PIB: Producto interno bruto

Fuente: autores

Para el 2009 la RT la región chilena de menor PIB, región de La Araucanía, egresaron del hospital por hidatidosis casi 28 veces más niños que la región de Antofagasta la cual dispone de mayor PIB per capita para ese año. A la vez, la DT entre estas dos regiones es de 14,7 por 100000 habitantes, lo cual significa en números absolutos, que en la región de La Araucanía hubo 44 egresos más que los que podrían esperarse si su situación mejorase hasta el nivel de la región de Antofagasta.

Para el 2010 la DT entre la región chilena con menor PIB per cápita, la región de La Araucanía, con la región de mejor PIB per cápita, región de Antofagasta, fue de 16,1 por 100.000 habitantes entre 0-18 años, lo cual significa en números absolutos, que en la región de La Araucanía hubo 48 egresos más de los que podrían esperarse si su situación económica mejorase hasta el nivel de la región de Antofagasta.

Para el 2010 y 2011 no se pudo obtener la RT dado que la región chilena de mejor PIB per capita no registró egresos hospitalarios para la hidatidosis. La DT entre la región con menor PIB per cápita, la región de La Araucanía, con la región de mejor PIB per cápita, región de Antofagasta, fue de 16,1 y 12,5 por 100.000 habitantes, para los años 2010 y 2011, respectivamente, lo cual significa en números absolutos, que en la región de La Araucanía hubo 48 y 37 egresos más de que los que podrían esperarse respectivamente si su situación mejorase hasta el nivel de la región de Antofagasta.

\section{RT Y DT SEGÚN IDH}

Para estas estimaciones se utilizó el IDH regional 2003.

Tabla 4. Razón de Tasas, Diferencia de Tasas y Números Absolutos de egresos de niños 0-18 años por hidatidosis según IDH, 2003

\begin{tabular}{|c|c|c|c|}
\hline Año & RT & DT & ${ }^{*} \mathbf{N}^{\circ}$ \\
\hline 2003 & 10,2 & 4,5 & 14,4 \\
\hline
\end{tabular}

*N: número absoluto de egresos

Fuente: autores

La Tabla 4 muestra que para el año 2003 la RT indica que en la región chilena de menor IDH, o situación socioeconómica, correspondiente a la región del Maule, los egresos hospitalarios correspondientes a esta región por hidatidosis son casi 10 veces más niños entre o y 18 años de edad que en la Región Metropolitana la cual dispone de mayor IDH, o mejor situación socioeconómica. A la vez, la DT entre estas dos regiones es de 4,5 por 100000 habitantes entre o y 18 años, lo cual significa en números absolutos, que en la región del Maule hubo 14 egresos más en menores entre o y 18 años que los que podrían esperarse si su situación mejorase hasta el nivel de la Región Metropolitana. 
mAYO - AGOSTO

\section{RIESGO ATRIBUIBLE POBLACIONAL PORCENTUAL (RAP\%)}

En la estimación del RAP\% se utilizaron las tasas del país y de las regiones chilenas con mejor situación económica (mejor PIB regional per cápita) y la de menor tasa de egresos hospitalarios. Sólo se consideraron las regiones que presentaron egresos hospitalarios. También fue estimado este indicador empleando el IDH 2003, único dato disponible para este indicador.

\section{RAP\% SEGÚN PIB PER CAPITA REGIONAL}

La Tabla 5 muestra que para el 2008 se podría haber evitado casi $81 \%$ de los egresos hospitalarios por hidatidosis atribuibles a la desventaja social, es decir, el $81 \%$ de los egresos hospitalarios se podrían evitar si se pudiese eliminar el efecto de la desventaja social en todos los grupos de la población chilena. Para igual año, pero según la menor tasa de egresos hospitalarios como referencia, se encuentra que cerca de $81 \%$ de los egresos por hidatidosis podrían ser evitados si fuera posible eliminar las múltiples exposiciones y vulnerabilidades asociadas a pertenecer a los diferentes grupos sociales.

En la Tabla 5 se muestran los demás valores donde destacan los años 2010 y 2011, donde según el PIB per capita regional se podría evitar en $100 \%$ los egresos hospitalarios si se pudiese eliminar el efecto de la desventaja social en todos los grupos de la población chilena. Si consideramos como referencia la menor tasa de egresos hospitalarios también se obtienen porcentajes entre $80,6 \%$ y $93,5 \%$.

\section{RAP\% SEGÚN IDH}

La Tabla 6 muestra que para el 2003 se podrían haber evitado casi $90 \%$ de los egresos hospitalarios por hidatidosis atribuibles a la desventaja social, medida por el IDH, es decir, el 90\% de los egresos hospitalarios se podrían evitar si se pudiese eliminar el efecto de la desventaja social en todos los grupos de la población.

\section{COEFICIENTE DE GINI Y CURVA DE LORENZ}

El resultado obtenido puede variar entre o y 1 . Donde 0 se interpreta como la ausencia de desigualdad y el valor 1 como la máxima desigualdad. Por lo tanto, mientras más se acerque al valor 1 mayor será la desigualdad en la distribución de los egresos hospitalarios.
En el estudio y según los datos de egresos hospitalarios por región para el 2011, se obtiene un Índice de Gini de 0,7 , es decir, una alta desigualdad en la distribución de los egresos hospitalarios por región.

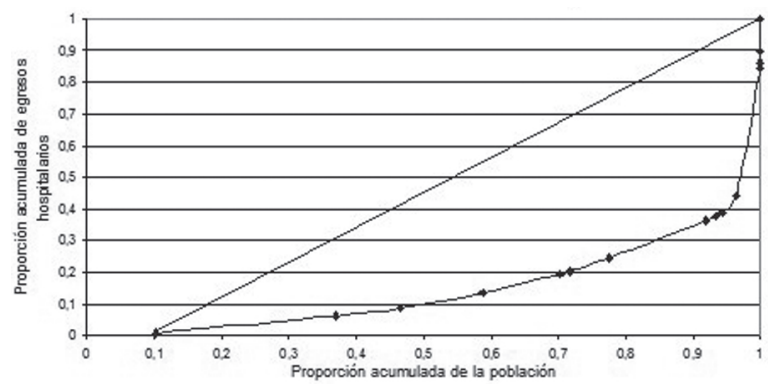

Figura 1: Curva de Lorenz según egresos hospitalarios de hidatiosis. Menores 0-18 años de edad. Chile, 2011

Fuente: Autores

Tabla 5. RAP\% de egresos de niños 0-18 años por hidatidosis de acuerdo a PIB per capita regional. 2008-2011

\begin{tabular}{|c|c|c|}
\hline & Mejor PIB & $\begin{array}{c}\text { Menor Tasa } \\
\text { Egresos }\end{array}$ \\
\hline Año & RAP\% & RAP\% \\
\hline 2008 & 80,6 & 80,6 \\
\hline 2009 & 85,7 & 88,3 \\
\hline 2010 & 100 & 93,5 \\
\hline 2011 & 100 & 90,8 \\
\hline
\end{tabular}

PIB: Producto interno bruto

Fuente: autores

Tabla 6. RAP\% egresos de niños 0-18 años por hidatidosis de acuerdo al IDH regional. 2003

\begin{tabular}{|c|c|c|}
\hline & Mejor PIB & $\begin{array}{c}\text { Menor Tasa } \\
\text { Egresos }\end{array}$ \\
\hline Año & RAP\% & RAP\% \\
\hline 2003 & 90,2 & 90,2 \\
\hline
\end{tabular}

Fuente: autores

\section{AÑOS DE VIDA POTENCIALMENTE PERDIDOS (AVPP)}

Entre 2001 y 2011 se registraron 458,81 años perdidos de vida por el fallecimiento precoz de 7 menores de edad, correspondiendo al impacto que produjo esta enfermedad sobre los años potenciales perdidos por muerte prematura. Los AVPP han disminuido 67,9\% entre el 2001 (AVPP = 207,22) y $2008($ AVPP $=66,45)$. Las mujeres (345,73 AVPP por 5 defunciones) perdieron más AVPP que los hombres (122,48 por 2 defunciones). Del total de grupos etario el orden de afección es: 10-14 años y 15-18 años (42,9\%); y 0-4 años (14,3\%). En hombres: $10-14$ años y 15-18 años 
(50\% respectivamente). En mujeres: 10-14 años y 15-18 años (40\%); y 0-4 años (20\%).

\section{Discusión}

Las limitaciones de este estudio radican en que se dispone de datos secundarios producto del sistema de vigilancia epidemiológica, y en el déficit de datos de los indicadores socioeconómicos, donde sólo se cuenta de datos para el PIB per capita regional para los años 2008 al 2011 y para el IDH' regional sólo se dispone de datos para el año 2003.

Los registros de casos ENO y egresos hospitalarios representan un porcentaje no despreciable en los menores entre o y 18 años. Si consideramos que del total de notificaciones ENO para todas las edades, un $21,4 \%$ corresponde a menores entre o y 18 años, mientras que para el registro de egresos hospitalarios es un $19,8 \%$.

La caracterización por distribución geográfica, nos muestra que la región chilena con mayor tasa de notificación ENO y egresos hospitalarios es Aysén. Sin embargo, destaca la región de la Araucanía con el mayor número absoluto tanto de casos ENO como egresos hospitalarios. Se ha de recordar que la región de la Araucanía presenta el PIB per cápita más bajo dentro del país, con los niveles más elevados de extrema pobreza y pobreza 5 .

Al comparar el tiempo de estadía con el registro nacional (todas las edades) no se observa diferencia significativa, encontrándose en los menores una estadía mediana de 8 días y en la población total una mediana de 9 días, lo que reafirma el avance médico/ tecnológico que permite resoluciones en menor tiempo y consecuentemente menor costo tanto económico como social5.

De acuerdo a los datos de mortalidad, se aprecia para el período un bajo número de muertes por esta causa e incluso no se registran defunciones desde el año 2008, lo que puede ser explicado por la eficiencia en los tratamientos una vez pesquisados los casos y la prioridad de atención a menores de edad ${ }^{6}$.

Según este estudio, el análisis de desigualdad realizada con los egresos hospitalarios, PIB e IDH para los años 2008 al 2011, dada la disponibilidad y limitación de datos, se observa consistentemente en cada año analizado que existe una diferencia importante en el número de egresos hospitalarios siendo mayor en la región chilena con menor PIB per capita y peor IDH. Por lo tanto, si existe un efecto de la gradiente social sobre la presentación de esta enfermedad, donde si se pudiesen igualar las condiciones socioeconómicas se podrían evitar entre 37 a 68 egresos. Para el análisis de RT, DT y número absoluto de egresos, considerando el PIB per capita regional la región chilena más desfavorecida fue la de La Araucanía. Mientras, al incluir el IDH 2003 la región más afectada fue la región del Maule. Se recuerda que el IDH tiene una mirada multidimensional que considera los aspectos de salud (tasa de AVPP), educación (alfabetismo de adultos, media escolaridad, cobertura educacional) e, ingresos (promedio del ingreso per cápita de los hogares, promedio ingreso per cápita de los hogares corregido por coeficiente de Gini). Y el propósito es responder a las necesidades de las personas y sociedades en términos de tener una vida sana y larga poseer los conocimientos necesarios para comprender y relacionarse reflexivamente con el entorno social y poseer los ingresos suficientes para acceder a un nivel de vida de mejor nivel.

Adicionalmente cuando se estima el RAP\% se encuentra que entre un $80 \%$ a $100 \%$ de los egresos hospitalarios por hidatidosis podrían ser evitados si fuese posible eliminar las múltiples exposiciones y vulnerabilidades asociadas a pertenecer a los diferentes grupos sociales.

En este estudio, la desigualdad encontrada en los análisis anteriores queda de manifiesto cuando se estima el coeficiente de Gini, obteniéndose un valor de 0,7 , lo cual dice que existe una alta desigualdad en los egresos hospitalarios, lo que significa que los egresos hospitalarios en menores entre 0-18 años de edad no se distribuyen homogéneamente entre las regiones.

La pérdida encontrada en este estudio de 458,81 años de vida por el fallecimiento prematuro de sólo 7 menores de edad es el impacto de esta enfermedad sobre la mortalidad en AVPP. No obstante, el bajo número de defunciones, se ha de considerar el impacto que tiene esta enfermedad en menores de edad, no sólo a nivel individual y familiar, sino también a nivel de sociedad, porque las muertes por hidatidosis en gente joven se relaciona a mayor pérdida de años de vida $y$, consecuentemente, mayor pérdida en años de productivos. Se ha de recordar que estas defunciones son muertes evitables, dado que es 
una enfermedad que tiene medidas de control, normas de diagnóstico y tratamiento ${ }^{6}$.

Adicionalmente, Chile ha ratificado la Convención de los Derechos del Niño, donde en su Artículo 24 hace referencia a la salud, donde los "Estados Partes reconocen el derecho del niño al disfrute del más alto nivel posible de salud"; Adoptar las medidas adecuadas para reducir la mortalidad infantil y en la niñez; Combatir las enfermedades aplicando la tecnología disponible y considerando los riesgos de contaminación del medio ambiente; Asegurar que todos los sectores de la sociedad, y en particular los padres y los niños, conozcan los principios básicos de la salud, la higiene y el saneamiento ambiental; y desarrollar la atención sanitaria preventiva, entre otros. De acuerdo a esta declaración cada vez que un menor de edad enferma o fallece a consecuencia de esta enfermedad absolutamente prevenible se ha de considerar como impedimento para el ejercicio de sus derechos, especialmente el derecho a la salud y consecuentemente a la vida"1.

Se hace necesario introducir en el equipo médico la importancia de la sospecha precoz. A la vez, es preciso considerar el estudio de los contactos de pacientes, especialmente de los menores de edad, con lo cual se puede acceder a un diagnóstico y tratamiento precoz. Lo anterior es relevante, toda vez que la literatura así lo reafirma. Por ejemplo, Anzieta et al., encontraron antecedentes epidemiológicos de familiares con previo diagnóstico de hidatidosis en aproximadamente $27 \%$ de los menores de 15 años de edad $^{12}$

En la actualidad se sabe que la hidatidosis es una enfermedad prevenible; se conocen los factores condicionantes y su forma de control, consecuentemente en Chile es factible su reducción, mediante acceso a la educación, información y participación de la población en las medidas de control y prevención en los diferentes grupos de riesgo; inspección sanitaria ganadero y fortalecimiento de los sistemas de vigilancia epidemiológica ${ }^{13}$. Consecuentemente, se hace necesario la evaluación del sistema actual de prevención, control y vigilancia, donde es preciso considerar una implementación de una política de control nacional, continua, a largo plazo, con enfoque multisectorial, interprogramático, que aborde los factores de riesgo y protección y, con disposición asegurada de recursos, lo cual implica necesariamente reconocer que es deber del Estado proteger la salud y alcanzar una mejor calidad de vida para toda la población, particularmente de los menores de edad. Por otro lado, para alcanzar éxito en la política, se debe integrar a los diferentes actores involucrados desde su generación hasta su evaluación, aprovechando las experiencias anteriores e infraestructura existente.

Finalmente, recordar que la infección se adquiere fundamentalmente en la niñez y los síntomas y sospecha clínica aparecen generalmente en los adultos. Sin embargo, cada nueva infección refleja el fracaso de las medidas de prevención y control y si es en menores -dada la edad- las podemos proyectar como un fracaso actual 6 .

\section{CONCLUSIÓN}

De acuerdo al análisis realizado en este estudio se puede evidenciar que existe un impacto de la gradiente social sobre la presentación de hidatidosis en menores de 18 años en Chile para el período en estudio, generando una desigualdad en la presentación de esta enfermedad. Por lo tanto, es necesario profundizar en estudios de detección precoz en menores de edad

\section{FinANCIAMIENTO}

El autor en este declara que este estudio se realizó sin aporte económico o becas. "Sin financiación".

\section{Conflicto de intereses}

No existe ningún tipo de relación financiera o personal que pudiesen dar lugar a conflicto de intereses con este artículo.

\section{REFERENCIAS BibLIOGRÁFICAS}

1. Rodulfo J, Carrión M, Freitas M, Real J, Merchán M. Hidatidosis pulmonar. Neumol Pediatr. 2013;8(1):5-9.

2. Mihmanli M, Idiz UO, Kaya C, Demir T, Bostanci O, Omeroglu S, et al. Estado actual del diagnóstico y el tratamiento de hepática equinococosis. J Hepatol Mundial. 2016;8(28):1169-1181.

3. Gobierno de Chile. Ministerio de Salud. Reglamento sobre notificación de enfermedades transmisibles de declaración obligatoria $\mathrm{N}^{\mathrm{0}}$ 158. Santiago, 22 de octubre de 2004.

4. Gobierno de Chile Ministerio de Salud. Normas Técnicas de Vigilancia de Enfermedades Transmisibles. [Internet] 2000 [citado 19 julio de 2014] Disponible en: http://epi.minsal.cl/epi/html/ public/enftransmisibles.pdf

5. Martínez P. Caracterización de la mortalidad por Hidatidosis Humana. Chile, 2000-2010. Rev Chil Infect. 2014;31(1):7-15

6. Martínez P. Factores climáticos, ambientales antropogénicos y socioeconómicos/demográficos, en la incidencia de hidatidosis en Chile (2001-2011): antecedentes para la formulación de políticas públicas de gestión ambiental en zoonosis [Tesis]. Chile: Universidad de Chile; 2017.

7. Schneider M, Castillo-Salado C, Bacallao J, Loyola E, Mujica O, 


\section{GALLEGOS-MARTÍIEZ PM}

Vidaurre M, et al. Métodos de medición de las desigualdades de salud. Rev Panam Salud Pública. 2002;12(6):398-415.

8. Frenz P, González C. Aplicación de una aproximación metodológica simple para el análisis de las desigualdades: El caso de la mortalidad infantil en Chile. Rev Med Chile. 2010;138:1157-1164.

9. Castillo-Salgado C, Schneider C, Loyola E, Mujica O, et al. Measuring Health Inequalities: Gini Coefficient and Concentration Index. Epidemiol Bull. 2001;22(1):3-4
MÉn.UIS. 2017-30(2)-29-3ь

10. Safaei J. Desigualdades en salud relacionadas con el ingreso global. Medicina Social. 2007;21(2):21-36

11. Convención sobre los Derechos del Niño Aprobada por la Asamblea General de las Naciones. Nuevo Siglo Editorial. 2006

12. Anzieta J, Caro M, Fierro C, Rocco E. Quiste hidatídico pulmonar en niños. Cuad. Cir. 2002;16:16-19.

13. Seremi Región del Bío Bio/ Servicio de Salud Bío Bío. Seminario "Actualizaciones en Hidatidosis". 26-27 junio de 2014. 